\title{
OPEN OXS2 is Required for Salt Tolerance Mainly through Associating with Salt Inducible Genes, CA1 and Araport11, in Arabidopsis
}

Ying Jing ${ }^{1,2,3}$, Lin $\mathrm{Shi}^{1,3}$, Xin $\mathrm{Li}^{1}$, Han Zheng ${ }^{1}$, Jianwei Gao ${ }^{1,2}$, Mei Wang ${ }^{1,2}$, Lilong $\mathrm{He}^{1,2^{*}}$ \& Wei Zhang ${ }^{1,2^{*}}$

Salt stress is one of the abiotic stresses affecting crop growth and yield. The functional screening and mechanism investigation of the genes in response to salt stress are essential for the development of salt-tolerant crops. Here, we found that OXIDATIVE STRESS 2 (OXS2) was a salinity-induced gene, and the mutant oxs2-1 was hypersensitive to salt stress during seed germination and root elongation processes. In the absence of stress, OXS2 was predominantly localized in the cytoplasm; when the plants were treated with salt, OXS2 entered the nuclear. Further RNA-seq analysis and qPCR identification showed that, in the presence of salt stress, a large number of differentially expressed genes (DEGs) were activated, which contain BOXS2 motifs previously identified as the binding element for AtOXS2. Further ChIP analysis revealed that, under salt stress, OXS2 associated with CA1 and Araport11 directly through binding the BOXS2 containing fragments in the promoter regions. In conclusion, our results indicate that OXS2 is required for salt tolerance in Arabidopsis mainly through associating with the downstream CA1 and Araport11 directly.

Salt stress, which is one of the representative abiotic stresses, reduces plant growth and crops yield worldwide ${ }^{1,2}$. As the overexploitation of farmland, salinization of soil is severely deteriorated. It has been reported that more than $30 \%$ of the farmland has been affected by salinity. By the year 2050, over half of farmland will be vulnerable to salt stress ${ }^{3}$. Salt stress increases soil osmotic potential, causes an imbalance of ions, disrupts physiological processes, inhibits plant growth and even leads to plant death ${ }^{4-10}$. Soil with a high concentration of salt reduces water absorption or even causes water extravasation from plant tissues, which leads to physiological drought, plasmolysis, and cell death ${ }^{10-13}$. High $\mathrm{Na}^{+}$and $\mathrm{Cl}^{-}$concentrations decrease enzyme activities in cells and thus restrict plant growth ${ }^{13-15}$. In addition, excess $\mathrm{Na}^{+}$and $\mathrm{Cl}^{-}$can lead to the accumulation of amino acids in plants and cause cell damage and death ${ }^{16}$. Under salt stress, the intracellular reactive oxygen species (ROS) are increased, which damages the plant cell plasma membrane, resulting in the loss of intracellular ions and organic matters ${ }^{17}$. ROS can also cause interference when external toxic ions penetrate into plant cells and inhibit plant growth and development ${ }^{16,18}$. Salt stress leads to a decreased photosynthesis in plants ${ }^{13}$. Low-concentration of salt promotes plant respiration, high-concentration of salt inhibits plant respiration, and excessive salt stress hinders plant protein synthesis ${ }^{19}$.

Salt tolerant crops can be achieved through two approaches. First, salt tolerant crops can be generated by normal hybridization breeding of existing salt tolerance genotypes ${ }^{20,21}$. Second, salt tolerance transgenic crops can be engineered through modifying functional salinity response genes ${ }^{22}$. For traditional breeding methods, the selection of stress tolerance traits is complicated and time-consuming, limiting the quality and the generation rate of tolerance varieties. On the contrary, genetic engineering technologies can provide new salt tolerance crops with specific functionalities in a much-shorter-period of time.

${ }^{1}$ Institute of Vegetables and Flowers, Shandong Key Laboratory of Greenhouse Vegetable Biology, Shandong Branch of National Vegetable Improvement Center, Huanghuai Region Vegetable Scientific Station of Ministry of Agriculture (Shandong), Shandong Academy of Agricultural Sciences, Jinan, 250100, China. ${ }^{2}$ Key Laboratory of Plant Development and Environment Adaptation Biology, Ministry of Education, School of Life Science, Shandong University, Qingdao, 266237, China. ${ }^{3}$ These authors contributed equally: Ying Jing and Lin Shi. *email: hllong1984@163.com; weizhang@sdu.edu.cn 


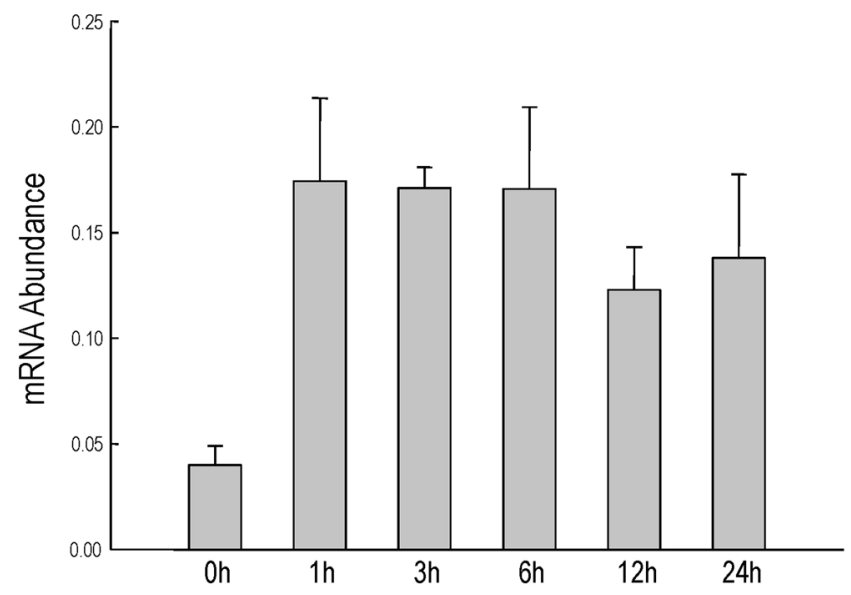

Figure 1. AtOXS2 transcript abundance in Arabidopsis without or with salt stress. AtOXS2 transcript abundance in Arabidopsis seedlings (relative to ACT2 control) determined by RT-qPCR. 10-day-old seedlings were exposed to $150 \mathrm{mM} \mathrm{NaCl}$. Error bars indicate \pm SD from three independent experiments.

OXIDATIVE STRESS 2 (OXS2), which belongs to a family containing five zinc finger (ZF) proteins with a canonical C2-H2 ZF and two C3-H ZFs, is a classical transcription factor ${ }^{23}$. OXS2 is widely expressed in plants, such as Arabidopsis, maize, rice, etc. Previously Arabidopsis OXS2 and two OXS2 homolog genes from maize ( $Z m O X S 2 b$ and $Z m O 2 L 1)$ have been found to play a role in stress escape and Cd stress tolerance ${ }^{24,25}$. In Arabidopsis, under high stress, AtOXS2 promotes plant stress escape by directly binding the promoter of SOC1, which is a representative floral transient gene ${ }^{24}$. In maize, the OXS2 homologous, $Z m O X S 2 b$ and $Z m O 2 L 1$, can confer Cd tolerance when heterogeneously expressed in Arabidopsis by activating the promoter of Cd-Inducible Methyltransferase 1 (CIMT 1), which is specifically expressed in root and also enhances the Cd resistant ability of Arabidopsis when overexpressed alone ${ }^{25}$. All of these three OXS2 members are able to directly recognize segments including the CT-rich BOXS2 motif. Here, we found that AtOXS2 was required for salt tolerance in Arabidopsis. RNA-seq analysis has selected multiply candidates which were controlled by AtOXS2 under salt stress. ChIP experiments suggest that AtOXS2 functions mainly through associating with the downstream salt induced and related genes, CA1 and Araport11, which are regulated by the BOXS2-containning promoters. Our findings suggest a new salt regulation mechanism, which can be potentially used for engineering salt tolerance in major crop plants.

\section{Results and Discussion}

AtOXS2 is inducible by salt in Arabidopsis. To test whether the expression of AtOXS2 is responsive to salt stress, Arabidopsis seedlings were grown in $1 / 2 \mathrm{MS}$ for $10 \mathrm{~d}$, and then transferred to the hydroponic culture plates with $150 \mathrm{mM} \mathrm{NaCl}$. The whole seedlings were collected at different time points after the salt treatment for quantitative reverse transcript PCR (RT-qPCR). The data indicated that the abundance of the AtOXS2 mRNA increased within $1 \mathrm{~h}$ after the salt treatment and stayed at a high level within the following $24 \mathrm{~h}$ (Fig. 1), indicating that the AtOXS2 transcript is activated in response to salinity stress and may be involved in plant salinity responses. As a classical transcription factor, AtOXS2 may control massive downstream cis-elements ${ }^{24}$. Under stress, the upregulation of the mRNA abundance will generate more proteins to activate genes which are related or resistant to the stress.

AtOXS2 is required for salt tolerance in Arabidopsis. To confirm if AtOXS2 is involved in plant salt tolerance, homozygotes of the T-DNA insertion mutant lacking detectable transcript by RT-PCR were collected for the salt stress test. As shown in Fig. 2(a-d), under the normal condition, the phenotype of the oxs2-1 plant was indistinguishable from that of the wild-type plants. In salt $(150 \mathrm{mM} \mathrm{NaCl})$ supplemented $1 / 2 \mathrm{MS}$ plates, the root length of oxs2-1 was shorter than that of the wild-type plants (Fig. 2(a,b)), and the shoot growth of oxs2-1 was also poorer than that of the wild-type plants (Fig. 2(c,d)). As germination is a key phenotype for plants to be resistant with salinity, germination rate tests were conducted in the mutants and the wild-type plants. As shown in Fig. 2(d), in the control environment, the germination rate of both oxs2-1 and wild-type plants had no obvious difference at $60 \mathrm{~h}$. However, in the presence of salinity, the germination rate of oxs $2-1$ was lower than that of the wild-type control after $48 \mathrm{~h}$. We also generated more than 5 independent OXS2-overexpressing (OE) transgenic lines, and two of these lines were randomly selected for the salt tolerance test, which were identified by RT-qPCR (Supplementary Fig. S1(a)). After three biological tests, we did not see any significant differential phenotype between the wild-type plants and the OE lines (Supplementary Fig. S1(b)). Although the OE lines did not show any significant salt tolerance phenotype, considering oxs2-1 is significantly salt sensitive, and AtOXS2: FLAG can recover the salt sensitive phenotype of oxs2-1 (Supplementary Fig. S2), we also conclude that OXS2 is required for salt tolerance in Arabidopsis. Considering the oxs2-1 plant is sensitive against diamide, and overexpressing OXS2 failed to yield plants with higher stress tolerance ${ }^{24}$, it is supposed that there exists a dose-effect for OXS2 to regulate stress tolerance in Arabidopsis, and excess OXS2 does not increase the contribution to stress tolerance. In a 


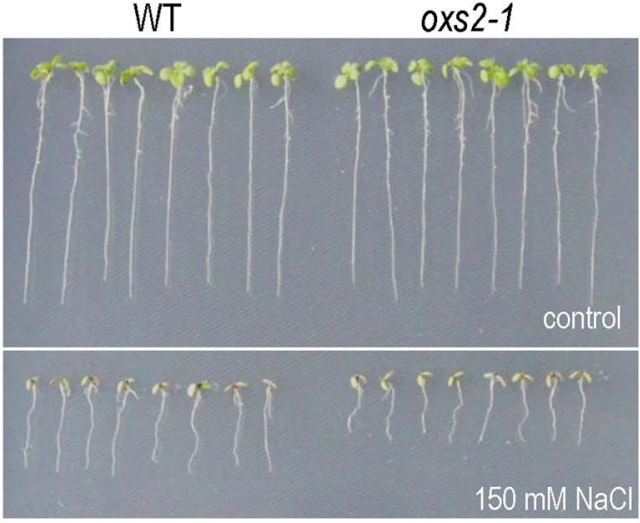

(a)

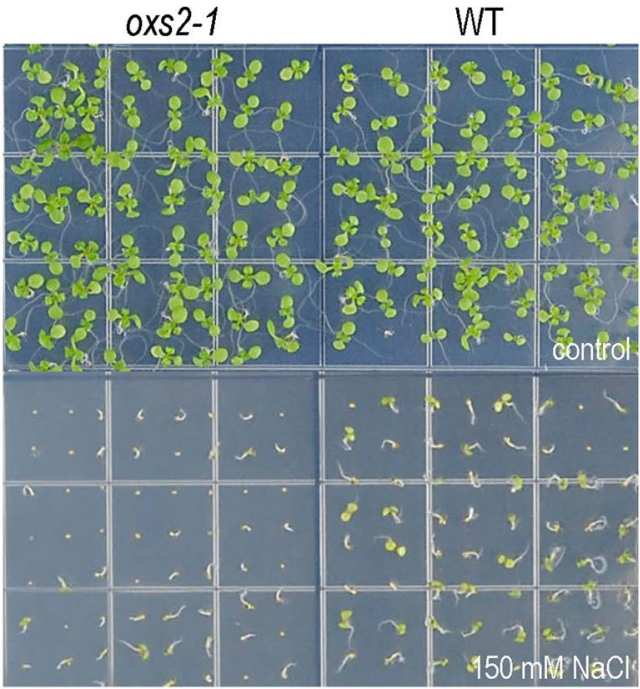

(c)

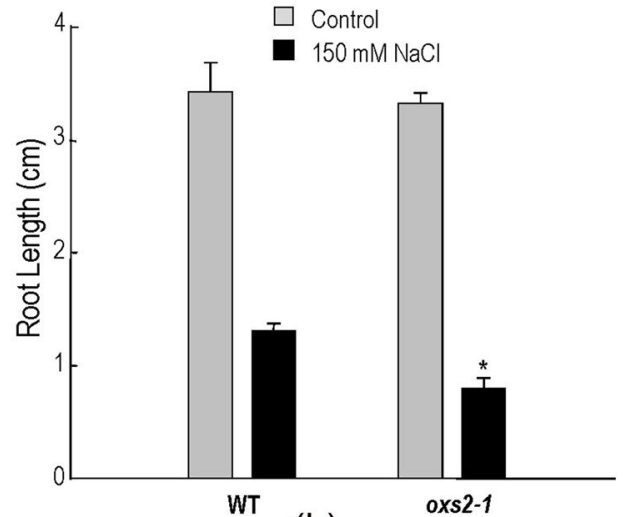

(b)

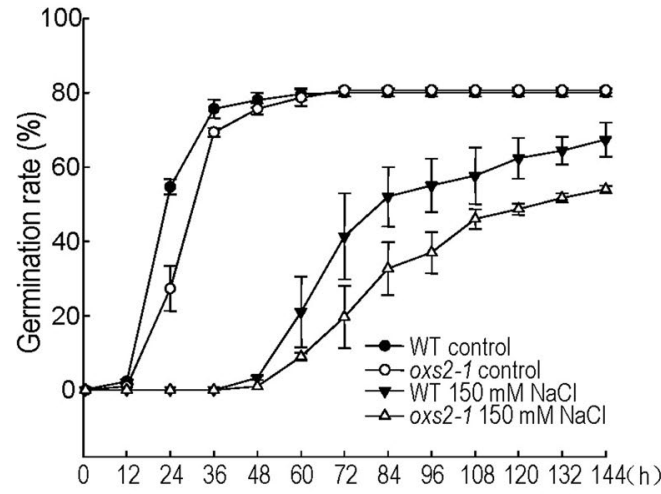

(b)

Figure 2. Phenotyping analysis of oxs2-1 and wild-type plants without or with salt stress. (a) Arabidopsis plants germinated on $1 / 2 \mathrm{MS}$ plates vertically for $3 \mathrm{~d}$ were transferred to plates without or with $150 \mathrm{mM} \mathrm{NaCl}$ for another $10 \mathrm{~d}$. Representative result from three reproducible experiments was shown. (b) Average root length of seedlings cultured as the same growth condition in (a). The root length of 5 seedlings of each class was measured as the mean value (remove the top and lowest value). Error bars indicates \pm SD from three independent experiments. (c) About 60 Arabidopsis seedlings were germinated and grown on $1 / 2$ MS plates horizontally without or with $150 \mathrm{mM} \mathrm{NaCl}$ for $10 \mathrm{~d}$. Representative test from three reproducible independent experiments was shown. (d) Germination rate of seedlings cultured as the same growth condition in (c). Error bars indicate + SD from three independent experiments.

word, comparing with the OE lines, the salt sensitive phenotype of the OXS2 mutant is more reliable for validating the function of OXS2. These results suggest that AtOXS2 plays a role in salt stress in Arabidopsis.

AtOXS2 is specifically accumulated in the nuclear under salt stress. AtOXS2 shows a canonical transcription factor feature and is accumulated in the nucleus under cold or ABA stress. However, there is no evidence supporting the translocation of AtOXS2 into the nuclear under salt stress. To test whether AtOXS2 plays a role as a transcription factor under salt stress, the coding region was fused to GFP expressed transiently in onion epidermal cells. GFP-Histone 4 (H4) specifically expressed in the nucleus was used as a positive control, and the empty vector (pGFP) was used as a negative control. In the absence of salt stress, the AtOXS2 fusion existed in the cytoplasm. However, when treated with $150 \mathrm{mM} \mathrm{NaCl}$, AtOXS2 was translocated into the nucleus, while the location of H4 or GFP was not affected by salt stress (Fig. 3). It is suggested that AtOXS2 specifically entered the nuclear under salt stress. The specific nuclear localization of AtOXS2 could play a role in salt tolerance at the molecular level. These results implied that AtOXS2 might target some downstream cis-elements which are required for salt stress responses in Arabidopsis.

Differentially regulated genes identified from RNA-Seq analysis. The salt-sensitive phenotype associated with the loss of AtOXS2 is likely due to the deletion of the transcription factor, AtOXS2 may recognize 


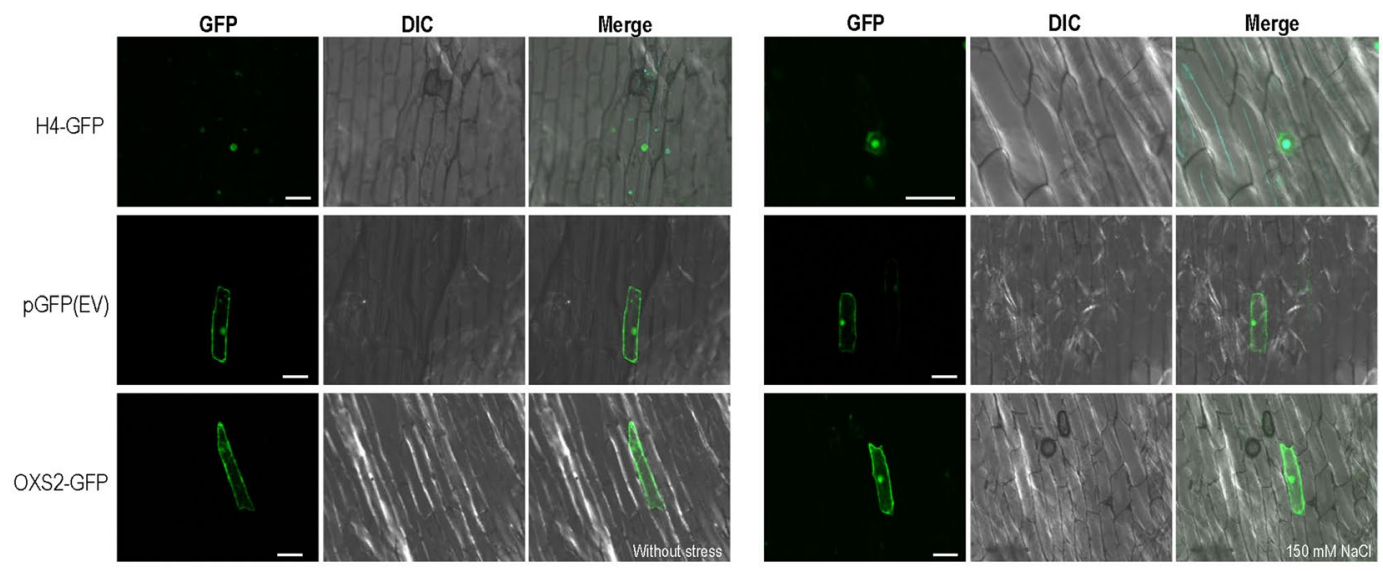

Figure 3. Subcellular localization of H4, OXS2 and empty vector tagged with GFP and transiently expressed in onion epidermal cells on $1 / 2 \mathrm{MS}$ for $24 \mathrm{~h}$ in the dark at room temperature and transfer to $1 / 2 \mathrm{MS}$ with $150 \mathrm{mM}$ $\mathrm{NaCl}$. The images were obtained from the GFP channel, DIC channel, or a merged image of the two channels. Scale bar: $100 \mu \mathrm{m}$.

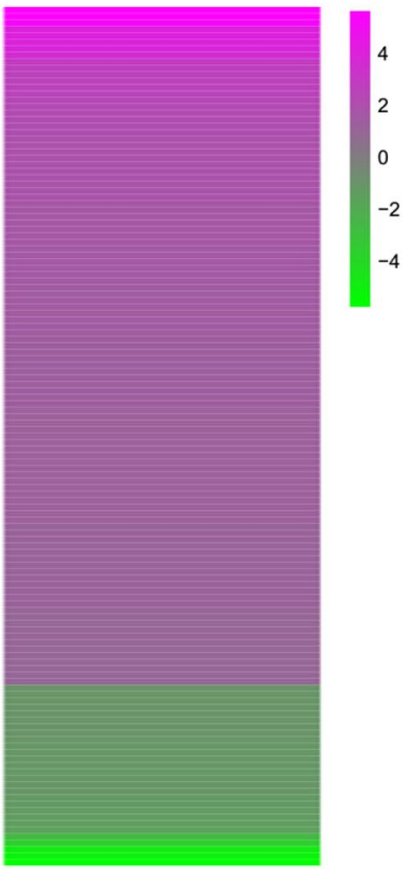

(a)

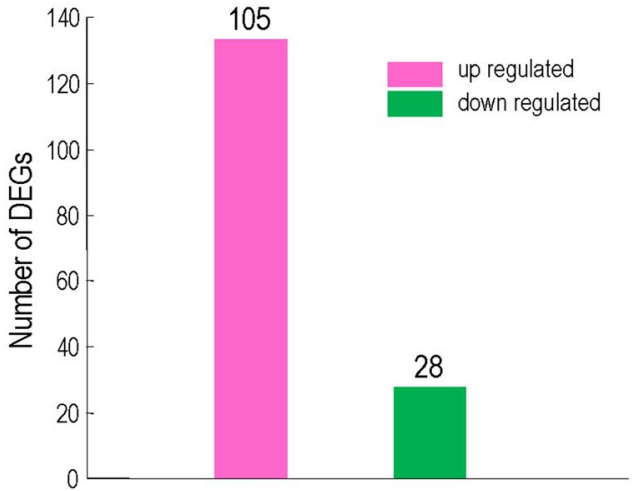

(b)

Figure 4. RNA-seq analysis. Heat map of clustering analysis of the 133 DEGs in oxs2-1 VS WT group. (a) Expression ratios shown as $\log 2$ values. Magenta represents increased expression; green represents decreased expression compared to oxs2-1. Vertical axis shows fold enrichment of relative transcript levels between oxs2-1 and wild-type plants. (b) Numbers of DEGs in in oxs2-1 VS WT group.

salt tolerance related genes from the protein and DNA interaction. To examine whether the gene expression pattern is affected, an RNA-seq analysis was carried out for the salt-treated oxs2-1 and wild-type plants. DEGs with statistically significant changes (up-regulated by at least 2 -fold or downregulated by at least 0.5 -fold, with a corrected P-Value $<0.05$ ) were selected. The total number of DEGs is 133 with 105 up-regulated DEGs and 28 down-regulated DEGs (Fig. 4(a,b)). Loss of AtOXS2 may lead to a decreased expression of the downstream genes. Thus, we narrowed the DEGs into 28 down-regulated DEGs, which were named from DEG1 to DEG 28 according to the RNA-seq ranking (Supplementary Table S1).

Validation of differential expression. To verify the expression pattern revealed by the RNA-seq analysis, qPCR was performed on the 27 down-regulated DEGs except DEG 9 (AtOXS2) with the same tissues used for RNA-seq. The expression pattern obtained by qPCR was not very consistent with the RNA-seq data, as the 


\begin{tabular}{|c|c|c|c|}
\hline $\begin{array}{l}\text { DEG } \\
\text { Number }\end{array}$ & GeneID & $\begin{array}{l}\text { Fold Change (qPCR) } \\
\text { WT VS oxs2-1 }\end{array}$ & BOXS2 \\
\hline DEG2 & AT3G44006 & 10.466369 & СТTCTТСТC (1386-1394) \\
\hline DEG3 & AT4G29200 & 10.464263 & СТTСТTCTC (1143-1151) \\
\hline DEG4 & AT4G34790 & 9.5341629 & CTTCCTTTC (892-900) \\
\hline DEG18 & AT4G12980 & 6.902787 & CTTCTTTTC (1609-1617) \\
\hline DEG10 & AT3G01500 & 5.0696121 & $\begin{array}{l}\text { CTCGCTCTC (1427-1435) } \\
\text { CTTCTTCTC (1525-1533) } \\
\text { CTTCTTTTC (1927-1935) }\end{array}$ \\
\hline DEG19 & AT5G25980 & 3.6420667 & \\
\hline DEG24 & AT5G38420 & 3.619712 & СТTСТТСТС (1089-1097) \\
\hline DEG5 & AT3G05945 & 3.2440316 & \\
\hline DEG6 & AT3G30720 & 3.0215489 & СТССТТTТС (1951-1959) \\
\hline DEG13 & AT3G27690 & 2.918036 & CTCGCTCTC (1022-1030) \\
\hline DEG12 & AT5G14740 & 2.7840692 & СТСССТTТC (931-939) \\
\hline DEG25 & AT1G29395 & 2.4340653 & \\
\hline DEG28 & AT3G05727 & 2.1557582 & СТTСТТСТС (1199-1207) \\
\hline DEG16 & AT4G14400 & 1.8827915 & \\
\hline DEG7 & AT2G42380 & 1.8514639 & СТTCTTCTC (153-161) \\
\hline DEG1 & AT3G49230 & 1.5516772 & \\
\hline DEG23 & AT1G11120 & 1.1261533 & СТТССТСТС (1938-1946) \\
\hline DEG22 & AT4G26530 & 1.0748932 & \\
\hline DEG15 & AT5G48490 & 1.044392 & \\
\hline DEG20 & AT4G02850 & 1.0277407 & CTCCTTGTC (1589-1597) \\
\hline DEG11 & AT2G34430 & 0.9983498 & \\
\hline DEG14 & AT2G29290 & 0.9854896 & \\
\hline DEG21 & AT2G34170 & 0.9825152 & \\
\hline DEG8 & AT5G38930 & 0.9305307 & \\
\hline DEG27 & AT1G62500 & 0.8855457 & \\
\hline DEG26 & AT1G73600 & 0.8696551 & \\
\hline DEG17 & AT1G10657 & 0.8609541 & CTTCTTTTC (174-182) \\
\hline DEG9 & AT2G41900 & & $\begin{array}{l}\text { CTCССТCTC (375-383) } \\
\text { СТССТTCTC (476-483) }\end{array}$ \\
\hline
\end{tabular}

Table 1. Summary of down-regulated differentially expressed genes in WT VS oxs2-1 comparison group discovered by RNA-seq. DEGs were ranked from top to bottom according to the fold change (WT VS oxs2-1) identified by qPCR. BOXS2 is a CT-rich motif in the putative promoter ( $2 \mathrm{~Kb}$ upstream of the coding region), which was calculated by a position frequency matrix tool according to the SAAB result ${ }^{24}$. Numbers in the parenthesis represent the position of the motif from $2 \mathrm{~Kb}$ before the coding region.

expression level of fourteen DEGs among the 27 down-regulated DEGs identified by RNA-seq did not decrease or decrease significantly (less than 2 fold). However, nearly half of the genes (13 DEGs) among the selected DEGs showed a dramatic change in gene expression (2.2- to 10.5-fold, Table 1). To explore the expression of the selected DEG expression in a greater detail, the expression level of the DEGs under the normal growth condition should also be detected. The mutants and wild-type plants were germinated on $1 / 2 \mathrm{MS}$ media for $3 \mathrm{~d}$, and then transferred to plates without or with $150 \mathrm{mM} \mathrm{NaCl}$ for another $10 \mathrm{~d}$ for RT-qPCR analysis of these thirteen dramatically decreased genes. As shown in Fig. 5, a number of salt-response genes were discovered. Some genes were over-expressed by salt stress in the wild-type plants, including DEG3, DEG6, DEG13, DEG18 and DEG19 (Fig. 5(b,e,h,i,j)). Among these genes, DEG6, DEG13 and DEG19 were not induced by salt stress in the absence of OXS2 (Fig. 5(b,h,j)), and the transcript abundance of DEG3 and DEG18 significantly decreased compared with that under the normal growth condition (Fig. 5(e,i)). It is suggested that OXS2 is the key upstream regulator of these five DEGs in response to salt stress. Besides the OXS2 related salt activated genes, some genes were down-regulated by salt stress in the wild-type plants, including DEG4, DEG10, DEG12, DEG24 and DEG28 (Fig. 5(c,f,g,k,m)). Four of these genes (DEG10, DEG12, DEG24 and DEG28) were expressed at a similar level without or with OXS2 under the normal growth condition, and DEG4 was significantly down-regulated in oxs2-1. However, all of these genes were dramatically down-regulated in the OXS2 mutants under salt stress (Fig. 5(c,f,g,k,m)). It is indicated that OXS2 also played an essential role as an upstream regulator in response to salt stress; without OXS2, the genes were more sensitive to salt stress. A number of genes (DEG2, DEG5 and DEG25) did not show a significant difference of the expression levels upon salt stress in the wild-type plants (Fig. 5(a,d,l)). However, in the OXS2 mutants, the expression of all of the DEGs was dramatically decreased (Fig. 5(a,d,l)). These results showed that, even in the absence of salt, all of these three genes were regulated by OXS2. 


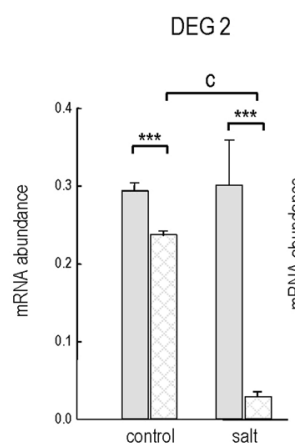

(a)

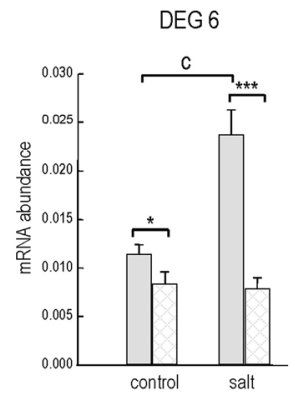

(e)

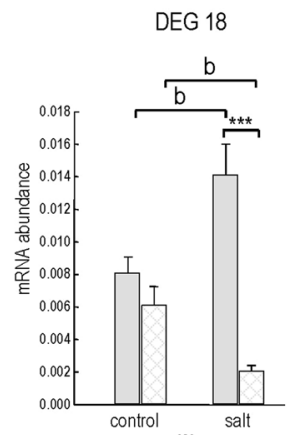

(i)

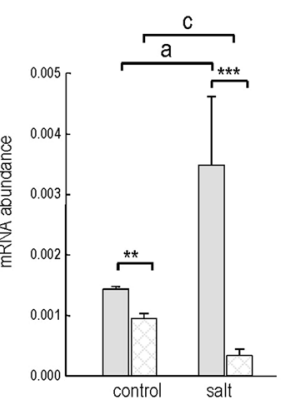

(b)

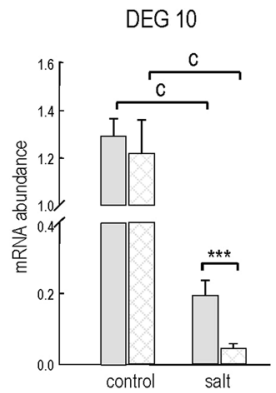

(f)

$\underline{\text { DEG } 19}$

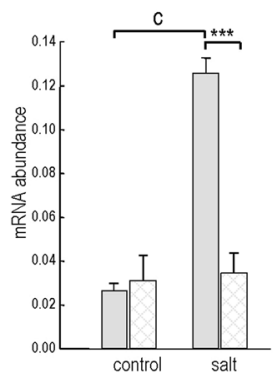

(j)

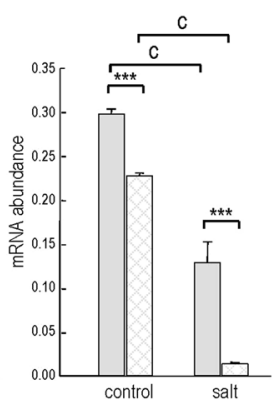

(c)

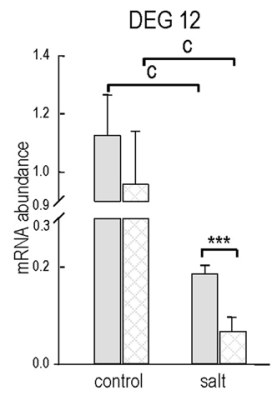

(g)

DEG 24

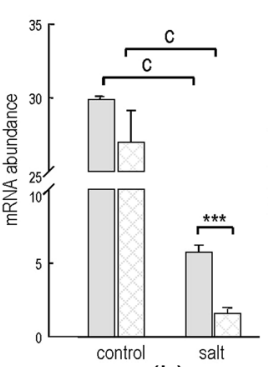

(k)
$\underline{D E G 5}$

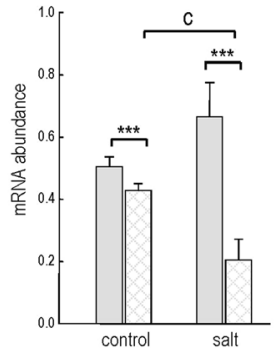

(d)

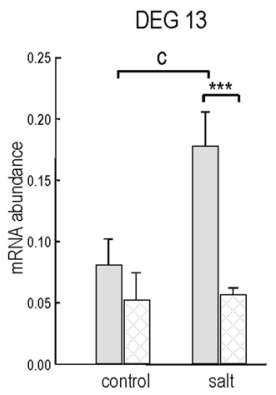

(h)

DEG 25

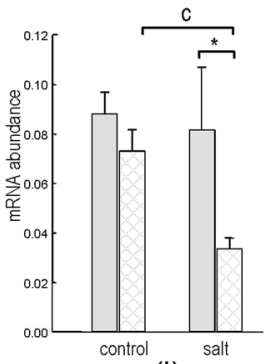

(I)

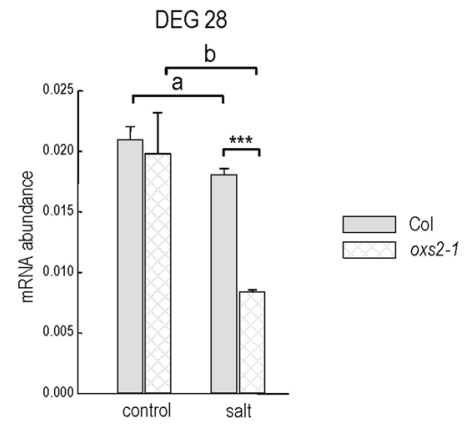

(m)

Figure 5. Expression of the selected DEGs (relative to ACT2) (a-m) in 13-d-old seedlings exposed to 0 or $150 \mathrm{mM} \mathrm{NaCl}$. Error bars indicate \pm SD from three independent experiments. P value of Student's $t$ test: wildtype compared with oxs $2-1$. ${ }^{*} \mathrm{P}<0.05 ; * \mathrm{P}<0.01$; $* * * \mathrm{P}<0.001$; Control compared with salt stress. ${ }^{\mathrm{P}} \mathrm{P}<0.05$; ${ }^{\mathrm{b}} \mathrm{P}<0.01{ }^{\mathrm{c}} \mathrm{P}<0.001$.

Salinity related pathways identified from RNA-Seq analysis. Salt-stress responses are always related to photosynthesis. Strong photosynthesis can enhance salt tolerance in some plant species. For example, increased photosynthesis confers salt stress tolerance in watermelon ${ }^{26}$. In our transcriptomic analysis, three of the 13 down-regulated DEGs are related to photosynthesis (Table 2). Salt stress also interrupts respiration and carbon metabolism in plants; efficient respiration is imperative for salinity tolerance ${ }^{15}$. Our analyses show that four of the 13 down-regulated DEGs are involved in carbon metabolism (Table 2). These results indicated that 
AtOXS2 might play a role in salt resistance through regulating photosynthesis and carbon metabolism. Salinity stress can induce oxidative damage in many plant species. Oxidative elements, such as ROS, play an important role in oxidative homeostasis and signaling pathways in response to salt stress ${ }^{27}$. As shown in Table 2 , two of the 13 DEGs are involved in the oxidation-reduction process. ABA-signaling is the central regulation pathway of salt-stress responses; salt stress treatment can be activated by ABA responses in plants ${ }^{28,29}$. In our results, two of the 13 down-regulated DEGs are involved in responses to ABA (Table 2). It is suggested that oxidative stress and $\mathrm{ABA}$ response related genes may also be regulated by AtOXS2.

Most significantly down-regulated DEGs are driven by cis-elements containing BOXS2 motifs. As a transcription factor, OXS2 mainly binds the DNA sequence containing a 9-bp CT rich motif, namely BOXS2, in vitro ${ }^{24}$. To predict the binding condition between OXS2 and the 27 selected genes in vivo, a computational framework for transcription factor binding site analysis (TFBS) was conducted using the Arabidopsis genome (Supplementary Table S2). As shown in Table 1, most genes with a dramatically decreased expression upon salt stress contained a BOXS2 motif except DEG5, DEG19 and DEG25. The DEGs with the most dramatic decrease of expression contained more BOXS2 motifs compared with the other genes. These results suggested that OXS2 regulated the downstream DEGs mainly through binding the BOXS2 motif. DEG5, DEG19 and DEG25, which do not contain a BOXS2 motif, were probably affected by other DEGs which were directly regulated by OXS2. Some of the putative OXS2-binding DEGs were constitutively activated by OXS2, including DEG2, DEG3, DEG4, and DEG6 (Fig. 5(a-c,e)). The others were activated by OXS2 under the treatment of salt, including DEG10, DEG12, DEG13, DEG18, DEG24 and DEG28 (Fig. 5(i,k,m)). Out of the three DEGs without BOXS2, DEG19 and DEG25 were involved in response to salt tolerance. Generally, at the molecular level, there are mainly two salt tolerance regulation manners. One is that the transcription factor constitutively binds the downstream salt tolerant DEGs without or with salt stress. The other is that, when the plant is treated with salt stress, the transcription factor expressed in the cytoplasm specifically enters the nuclear and activates other downstream salt tolerant DEGs.

AtOXS2 associates with CA1 and Araport11 directly. To explore the interaction between the BOXS2-containing promoters and AtOXS2, we generated transgenic Arabidopsis oxs2-1 producing an FLAG-tagged AtOXS2, which can recover the salt sensitive phenotype (Supplementary Fig. S2). After identifying the expression of the fusion protein by western blot (Supplementary Fig. S3), a chromatin immunoprecipitation-quantitative PCR (ChIP-qPCR) analysis was performed to test the in vivo interaction of these promoters in oxs2-1 and oxs2-1 (AtOXS2-FLAG). Following immunoprecipitation with anti-FLAG antibody, twelve pairs of primers were used for the ten promoters corresponding to fragments F1-F12 (Fig. 6). Positive interaction with AtOXS2 was found for F4 and F7 (Fig. 6), but not for the other fragments, including the ACT2 (At3g18780) promoter used as the negative control. It is indicated that AtOXS2 could bind the promoters of DEG10 (carbonic anhydrase 1, CA1) and DEG18 (Auxin-responsive family protein gene, Araport11) directly. CA1 is induced by salt stress in Dunaliella salina, and the promoter of CA1 is able to drive a stable expression of two foreign genes (BAR and GUS) in transformed cells of $D$. salina under salt stress ${ }^{30}$. Araport11 (NAC domain containing protein 1) is a member of the NAC transcription factor family. Araport11 of Suaeda liaotungensis $\mathrm{K}$ (SlNAC1) can enhance salt tolerance in Arabidopsis ${ }^{31}$. The other fragments of promoters were possibly regulated through the cis-elements of AtOXS2 or unknown interactive proteins of the transcript factors.

\section{Conclusion}

Although OXS2 was proved to be responsive to several types of stresses, such as ABA, cold, diamide and $\mathrm{Cd}^{24,25}$, there is no report indicating that OXS2 was involved in salt stress. In this paper, we found the loss of OXS2 led to a salt-sensitive phenotype of Arabidopsis with multiple disrupted pathways or molecular functions. Further analysis identified that OXS2 regulated salt tolerance mainly through associating with BOXS2 motif containing DEGs, such as CA1 and Araport11, which are related to salt responses. We found that both of DEG10 and DEG18 were down regulated in oxs2-1 under salt stress (Fig. 5(f,i)). These DEGs are different from the constitutively down-regulated DEGs, such as DEG2, DEG3, DEG4, DEG5 and DEG6 (Fig. 5(a-e)), which showed a decreased level in oxs2-1 under the normal growth condition. It is consistent with the protein localization of OXS2 without or with salt stress (Fig. 3). These results suggest that the salt tolerance regulated by OXS2 was specifically induced by salt stress in Arabidopsis. Without stress, OXS2 localized in cytoplasmic; when treated with salt, OXS2 entered the nuclear and associated with the promoters of CA1 and Araport11, and played a role in salt tolerance in Arabidopsis (Fig. 7).

\section{Methods}

Plant culture and treatment. Arabidopsis thaliana, wild-type Col-0 (SALK_6000) and T-DNA insertion mutant oxs2-1 (SALK_037470) have been described previously ${ }^{24}$. Arabidopsis plants were grown in a controlled environment at $22^{\circ} \mathrm{C} / 20^{\circ} \mathrm{C}$ in a 16 -h-light $/ 8$-h-dark photoperiod. Seeds used for phenotypic assays were harvested at the same time. The tolerance test was performed on plates with $1 / 2$ MS solid media without or with $150 \mathrm{mM} \mathrm{NaCl}$. The cultured seeds were germinated in plates without stress for $3 \mathrm{~d}$, and then transferred to plates with $150 \mathrm{mM} \mathrm{NaCl}$ for another $10 \mathrm{~d}$.

For the salt-inducible experiment, the Arabidopsis plants were germinated and grown in $1 / 2 \mathrm{MS}$. Ten-day-old seedlings were transferred to $1 / 2 \mathrm{MS}$ hydroponic cultures with $150 \mathrm{mM} \mathrm{NaCl}$, and 10 seedlings were collected at different time points from the start of the salt treatment. 


\begin{tabular}{|c|c|c|c|c|c|c|}
\hline $\begin{array}{l}\text { DEG } \\
\text { Number }\end{array}$ & GeneID & $\begin{array}{l}\log _{2} \text { Ratio WT } \\
\text { VS oxs2-1 }\end{array}$ & $\begin{array}{l}\text { Fold Change } \\
\text { WT VS } \\
\text { oxs2-1 }\end{array}$ & & $\begin{array}{l}\text { Results of blast against nr } \\
\text { database }\end{array}$ & TAIR Annotation (Key Words) \\
\hline DEG2 & AT3G44006 & -5.2148 & 0.026927463 & & hypothetical protein & $\begin{array}{l}\text { This gene encodes a small protein and } \\
\text { has either evidence of transcription or } \\
\text { purifying selection. }\end{array}$ \\
\hline DEG3 & AT4G29200 & -4.8655 & 0.034304627 & & $\begin{array}{l}\text { Beta-galactosidase related } \\
\text { protein }\end{array}$ & Beta-galactosidase related protein \\
\hline DEG4 & AT4G34790 & -3.6286 & 0.080848939 & & $\begin{array}{l}\text { SAUR-like auxin-responsive } \\
\text { protein family }\end{array}$ & response to auxin \\
\hline DEG5 & AT3G05945 & -2.5701 & 0.168388441 & & & \\
\hline DEG6 & AT3G30720 & -1.5808 & 0.334298315 & & qua-quine starch & $\begin{array}{l}\text { negative regulation of starch metabolic } \\
\text { process, positive regulation of protein } \\
\text { metabolic process, starch biosynthetic } \\
\text { process }\end{array}$ \\
\hline DEG10 & AT3G01500 & -1.3002 & 0.406068198 & $\alpha, \beta$ & carbonic anhydrase 1 & $\begin{array}{l}\text { carbon utilization, defense response } \\
\text { to bacterium, defense response to } \\
\text { fungus, incompatible interaction, } \\
\text { negative regulation of stomatal } \\
\text { complex development, photosynthesis, } \\
\text { regulation of stomatal movement, } \\
\text { response to carbon dioxide, response } \\
\text { to cold }\end{array}$ \\
\hline DEG12 & AT5G14740 & -1.1985 & 0.435715601 & $\beta$ & carbonic anhydrase 2 & $\begin{array}{l}\text { carbon utilization, defense response } \\
\text { to bacterium, regulation of stomatal } \\
\text { movement, response to carbon dioxide }\end{array}$ \\
\hline DEG13 & AT3G27690 & -1.1934 & 0.437262295 & $\alpha$ & $\begin{array}{l}\text { photosystem II light } \\
\text { harvesting complex protein } \\
2.3\end{array}$ & $\begin{array}{l}\text { response to blue light, response to } \\
\text { cold, response to desiccation, response } \\
\text { to far red light, response to high light } \\
\text { intensity, response to light stimulus, } \\
\text { response to low light intensity stimulus, } \\
\text { response to red light }\end{array}$ \\
\hline DEG18 & AT4G12980 & -1.1046 & 0.465026703 & $x$ & $\begin{array}{l}\text { Auxin-responsive family } \\
\text { protein }\end{array}$ & $\begin{array}{l}\text { multicellular organism development, } \\
\text { oxidation-reduction process }\end{array}$ \\
\hline DEG19 & AT5G25980 & -1.0972 & 0.467436341 & $\beta, \delta$ & glucoside glucohydrolase 2 & $\begin{array}{l}\text { abscisic acid-activated signaling } \\
\text { pathway, carbohydrate metabolic } \\
\text { process, defense response to insect, } \\
\text { glucosinolate catabolic process, } \\
\text { regulation of stomatal movement, } \\
\text { response to abscisic acid, response to } \\
\text { salt stress }\end{array}$ \\
\hline DEG24 & AT5G38420 & -1.0376 & 0.487144309 & $\alpha, \beta$ & $\begin{array}{l}\text { Ribulose bisphosphate } \\
\text { carboxylase (small chain) } \\
\text { family protein }\end{array}$ & $\begin{array}{l}\text { carbon fixation, oxidation-reduction } \\
\text { process, photorespiration, reductive } \\
\text { pentose-phosphate cycle, response to } \\
\text { blue light, response to far red light, } \\
\text { response to red light }\end{array}$ \\
\hline DEG25 & AT1G29395 & -1.0314 & 0.489218757 & $\delta$ & $\begin{array}{l}\text { COLD REGULATED } 314 \\
\text { INNER MEMBRANE } 1\end{array}$ & $\begin{array}{l}\text { cellular response to cold, cellular } \\
\text { response to water deprivation, cold } \\
\text { acclimation, response to abscisic acid }\end{array}$ \\
\hline DEG28 & AT3G05727 & -1.0069 & 0.497612635 & & $\begin{array}{l}\text { S locus-related glycoprotein } \\
1 \text { (SLR1) binding pollen } \\
\text { coat protein family }\end{array}$ & $\begin{array}{l}\text { defense response to fungus, killing of } \\
\text { cells of other organism }\end{array}$ \\
\hline
\end{tabular}

Table 2. Summary of down-regulated differentially expressed genes in WT VS oxs2-1 comparison group identified by qPCR (less than 2 fold). $\alpha$ : description of photosynthesis, $\boldsymbol{\beta}$ : description of carbon metabolism, $\chi$ : description of oxidation-reduction process, $\delta$ : description of response to ABA.

Seed germination bioassay. Arabidopsis seeds were planted in $1 / 2 \mathrm{MS}$ and placed at $4{ }^{\circ} \mathrm{C}$ for $3 \mathrm{~d}$. Emerge-germinating seeds were counted in a controlled environment at $22^{\circ} \mathrm{C} / 20^{\circ} \mathrm{C}$ in a 16 -h-light/8-h-dark photoperiod at different time points.

Molecular constructs. For the transient expression construct, the AtOXS2 coding region without the stop codon was PCR amplified from Arabidopsis (Col) cDNA with the primer pair of AtOXS2 F: $5^{\prime}$ GCGTCGACATGTGCTGTGGATCAGACC $3^{\prime}$ and AtOXS2 R: $5^{\prime}$ CGGGATCCTCAATTCTGCTGAGCCACA $3^{\prime}$. The amplified fragment was inserted into the transient expression vector pGFP using the SacI and BamHI sites. For the expression construct of AtOXS2, the coding region was PCR amplified from Arabidopsis (Col) cDNA with the primer sets AtOXS2-1F/AtOXS2-1R. The DNA was inserted into the XbaI site of the binary vector pCambia3300 (http://www.cambia.org) to yield p35S::AtOXS2 using the In-Fusion HD cloning kit (catalog No. 011614; Clontech). To make the 35S::AtOXS2-FLAG construct, the AtOXS2 coding region without its stop codon was PCR amplified from genomic DNA with the primers AtOXS2-2F/AtOXS2-R and inserted between the KpnI and PstI sites on pCambia1305. All primers are listed in Supplementary Table S3.

Protein subcellular localization assay. Transient expression in onion epidermal cells was carried out using the Biolistic PDS 1000/He Particle Delivery System (Bio-Rad) (1100 psi, $10 \mathrm{~cm}$ traveling distance) with 


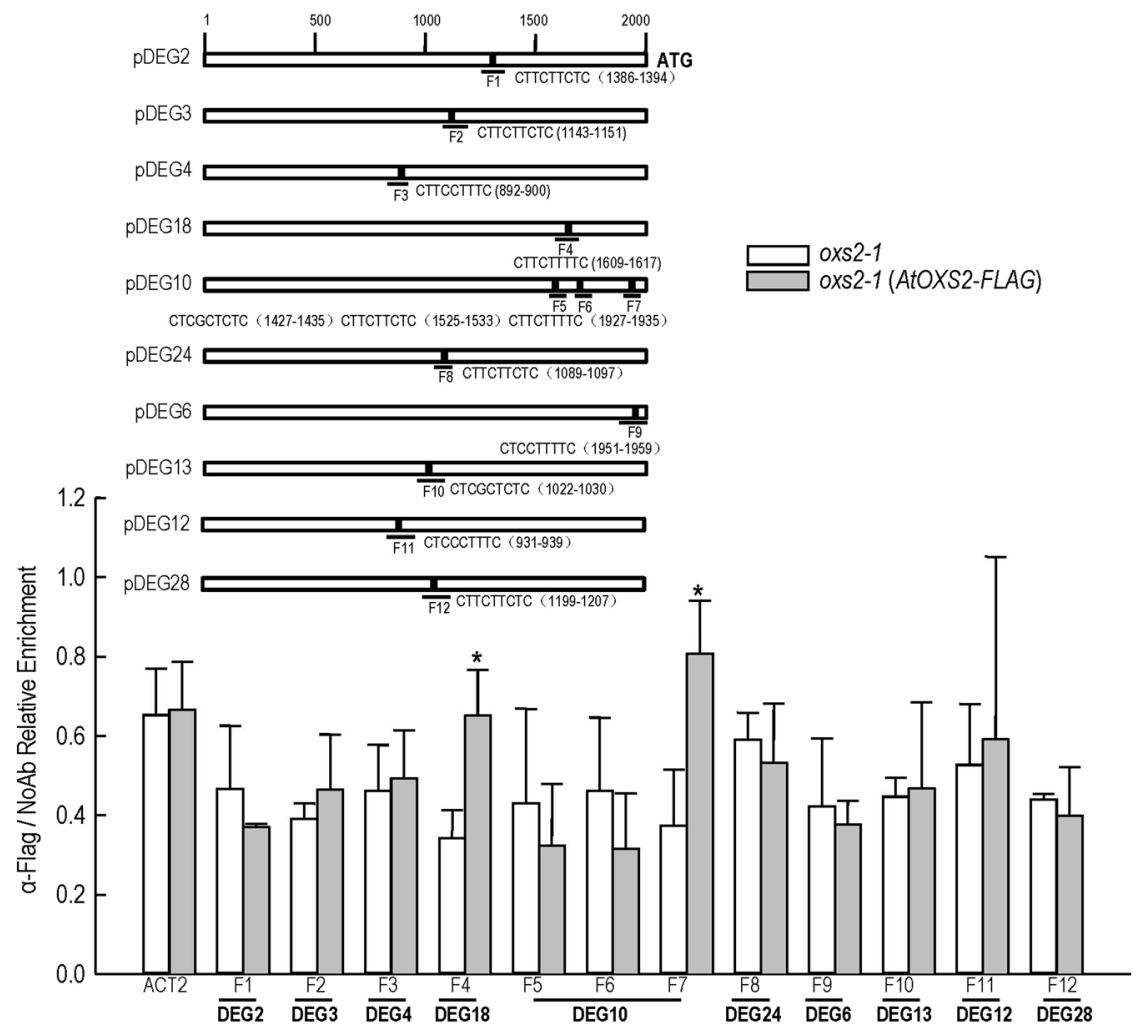

Figure 6. ChIP-qPCR to test in vivo interaction of promoters (including 5'UTR) with AtOXS2 in 10-d-old seedlings from oxs2-1 and oxs2-1 (AtOXS2-FLAG) treated with $150 \mathrm{mM} \mathrm{NaCl}$. Promoter or segments tested are labeled F1-F12. CP (crossing point) value of immuno-precipitated DNA fractions with $\alpha$-FLAG or no antibody control (NoAb) normalized to CP value of input DNA fractions for the same qPCR assay. Y axis is the ChIP signals calculated as the enrichment relative to the no-antibody control (No Ab). Error bars indicate \pm SD from three independent experiments. P value of Student's t test: oxs2-1 (AtOXS2-FLAG) compared with oxs2-1. $* \mathrm{P}<0.05 ; * * * \mathrm{P}<0.001$.

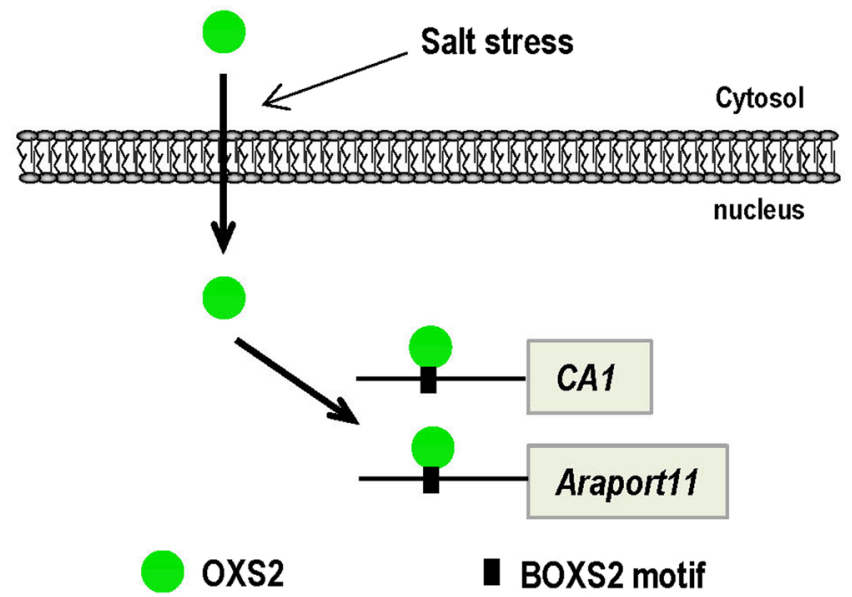

Figure 7. Model of OXS2 regulation of salt stress.

plasmid DNA, $0.5 \mathrm{M} \mathrm{CaCl}_{2}$ and $10 \mathrm{mM}$ spermidine precipitated onto $1 \mathrm{~mm}$ gold particles. Plasmids were delivered onto onion epidermis cultured by $1 / 2 \mathrm{MS}$ without or with $150 \mathrm{mM} \mathrm{NaCl}$. After incubating for about $10 \mathrm{~h}$ in the dark at $25^{\circ} \mathrm{C}$, the epidermis was observed under a Zeiss 300 confocal microscope.

RT-qPCR. RNA extraction was conducted using a plant RNA kit (catalog No. R5105; GBCBIO Technologies). Reverse transcription was conducted using PrimeScript RT reagent kit with a gDNA Eraser (catalog No. RR047A; TaKaRa). qPCR was conducted with SYBR Premix Ex Taq (catalog No. DRR820A; TaKaRa) on a LightCycler 480 
II (Roche). Arabidopsis ACT2 (AT3G18780) was used as an internal control, and the $2^{-\Delta \Delta C T}$ method was used in the analysis of the real time PCR data. All primers are listed in Supplementary Table S3.

RNA-Seq library construction and sequencing. The wild-type plants and oxs $2-1$ were germinated on $1 / 2$ MS plates for $3 \mathrm{~d}$, and then transferred to plates with $150 \mathrm{mM} \mathrm{NaCl}$ for another $10 \mathrm{~d}$. Three independent batches of the 10-d-old plantlets were collected, and then stored in $-80^{\circ} \mathrm{C}$ before sending to Novogene for RNA-seq analysis. Total RNA isolation, library construction, sequencing, and basic data analysis were carried out by Novogene.

Western blot. Arabidopsis leaves were harvested and ground with $2 \mathrm{~mL}$ of immunoprecipitation buffer $(50 \mathrm{mM}$ TrisHCl, pH 7.5, $150 \mathrm{mM} \mathrm{NaCl}$, and $1 \%$ [v/v] Triton X-100) with freshly prepared DTT (1 mM) and 1X protease inhibitor cocktail (catalog No. 04693132001; Roche) on ice. Protein extracts were centrifuged at $12,000 \mathrm{~g}$ for $30 \mathrm{~min}$ at $4{ }^{\circ} \mathrm{C}$. A total of $100 \mu \mathrm{L}$ of supernatant was stored at $-80^{\circ} \mathrm{C}$ for immunoblot. Protein samples $(20 \mu \mathrm{L})$ were loaded onto $10 \%(\mathrm{w} / \mathrm{v})$ SDS-PAGE gels and the immunoblot was conducted as described before ${ }^{32}$.

Chromatin immunoprecipitation. Arabidopsis seeds were germinated in $1 / 2 \mathrm{MS}$ for $3 \mathrm{~d}$, and then the same size seedlings were transferred to the $1 / 2 \mathrm{MS}$ with $150 \mathrm{mM} \mathrm{NaCl}$ and cultured for another $7 \mathrm{~d}$. About one gram fresh tissue (whole seedlings) was collected to perform chromatin immunoprecipitation as described before $\mathrm{e}^{25}$. All primers are listed in Supplementary Table S3.

Accession numbers. Sequence data for the RNA-seq samples can be found in the NCBI's Sequence Read Archive (SRA) database under the following accession number: SUB4904863.

Received: 6 August 2019; Accepted: 6 December 2019;

Published online: 30 December 2019

\section{References}

1. Munns, R. Comparative physiology of salt and water stress. Plant Cell Environ. 25, 239-250 (2002).

2. Rengasamy, P. World salinization with emphasis on Australia. J. Exp. Bot. 57, 1017-1023 (2006).

3. Wang, W. X., Vinocur, B. \& Altman, A. Plant responses to drought, salinity and extreme temperatures: towards genetic engineering for stress tolerance. Planta. 218, 1-14 (2003).

4. Glenn, E. P., Brown, J. J. \& Blumwald, E. Salt tolerance and crop potential of halophytes. Critical Reviews in Plant Sciences. 18, 227-255 (1999)

5. Sairam, R. K. \& Tyagi, A. Physiology and molecular biology of salinity stress tolerance in plants. Curr. Sci. 86, 407-421 (2004).

6. Munns, R. \& Tester, M. Mechanisms of salinity tolerance. Annu. Rev. Plant Biol. 59, 651-681 (2008).

7. Shi, H. Z., Lee, B. H., Wu, S. J. \& Zhu, J. K. Overexpression of a plasma membrane $\mathrm{Na}+/ \mathrm{H}+$ antiporter gene improves salt tolerance in Arabidopsis thaliana. Nature biotechnology. 21, 81-85 (2003).

8. Munns, R. Genes and salt tolerance: bringing them together. New Phytol. 167, 645-663 (2005).

9. Tuteja, N. Mechanisms of high salinity tolerance in plants. In Osmosensing and Osmosignaling, Haussinger, D.; Sies, H., Eds. Elsevier Academic Press Inc: San Diego. 428, 419-438 (2007).

10. Ismail, A., Takeda, S. \& Nick, P. Life and death under salt stress: same players, different timing? J. Exp. Bot. 65, 2963-2979 (2014).

11. Khan, M. A., Ungar, I. A. \& Showalter, A. M. Effects of salinity on growth, water relations and ion accumulation of the subtropical perennial halophyte, Atriplex griffithii var. stocksii. Annals of Botany. 85, 225-232 (2000)

12. Shavrukov, Y. Salt stress or salt shock: which genes are we studying? J. Exp. Bot. 64, 119-127 (2013).

13. Chaves, M. M., Flexas, J. \& Pinheiro, C. Photosynthesis under drought and salt stress: regulation mechanisms from whole plant to cell. Annals of Botany. 103, 551-560 (2009).

14. Wang, W. B. et al. Analysis of antioxidant enzyme activity during germination of alfalfa under salt and drought stresses. Plant Physiology and Biochemistry. 47, 570-577 (2009)

15. Jacoby, R. P., Taylor, N. L. \& Millar, A. H. The role of mitochondrial respiration in salinity tolerance. Trends Plant Sci. 16, 614-23 (2011).

16. Flowers, T. J., Munns, R. \& Colmer, T. D. Sodium chloride toxicity and the cellular basis of salt tolerance in halophytes. Annals of Botan. 115, 419-431 (2015).

17. Leshem, Y., Seri, L. \& Levine, A. Induction of phosphatidylinositol 3-kinase-mediated endocytosis by salt stress leads to intracellular production of reactive oxygen species and salt tolerance. Plant Journal. 51, 185-197 (2007).

18. Tattini, M. et al. Olea-Europaea Plants During Salinity Stress And Subsequent Relief. Physiol. Plant. 95, 203-210 (1995).

19. Yang, H. B. Effects of osmotic and salt stress on some physiological indexes of wheat seedlings. Res. Crop. 14, 404-408 (2013).

20. Lisa, L. A. et al. Physiology and gene expression of the rice landrace Horkuch under salt stress. Functional Plant Biology. 38, 282-292 (2011).

21. Hanin, M., Ebel, C., Ngom, M., Laplaze, L. \& Masmoudi, K. New Insights on Plant Salt Tolerance Mechanisms and Their Potential Use for Breeding. Frontiers in Plant Science. 7, 1787 (2016).

22. Kasuga, M., Liu, Q., Miura, S., Yamaguchi-Shinozaki, K. \& Shinozaki, K. Improving plant drought, salt, and freezing tolerance by gene transfer of a single stress-inducible transcription factor. Nature biotechnology. 17, 287-291 (1999).

23. Mosavi, L. K., Cammett, T. J., Desrosiers, D. C. \& Peng, Z. Y. The ankyrin repeat as molecular architecture for protein recognition. Protein Science. 13, 1435-1448 (2004).

24. Blanvillain, R., Wei, S., Wei, P., Kim, J. H. \& Ow, D. W. Stress tolerance to stress escape in plants: role of the OXS2 zinc-finger transcription factor family. The EMBO journal. 30, 3812-3822 (2011).

25. He, L. et al. Maize OXIDATIVE STRESS2 Homologs Enhance Cadmium Tolerance in Arabidopsis through Activation of a Putative SAM-Dependent Methyltransferase Gene. Plant Physiol. 171, 1675-1685 (2016).

26. Li, H. et al. Exogenous Melatonin Confers Salt Stress Tolerance to Watermelon by Improving Photosynthesis and Redox Homeostasis. Front Plant Sci. 8, 295 (2017).

27. Miller, G., Suzuki, N., Ciftci-Yilmaz, S. \& Mittler, R. Reactive oxygen species homeostasis and signalling during drought and salinity stresses. Plant Cell Environ. 33, 453-467 (2010).

28. Zhu, J. K. Abiotic Stress Signaling and Responses in Plants. Cell. 167, 313-324 (2016).

29. Zhu, J. K. Salt and drought stress signal transduction in plants. Annu. Rev. Plant Biol. 53, 247-273 (2002).

30. Li, J., Lu, Y., Xue, L. \& Xie, H. A structurally novel salt-regulated promoter of duplicated carbonic anhydrase gene 1 from Dunaliella salina. Mol Biol Rep 37, 1143-1154, https://doi.org/10.1007/s11033-009-9901-z (2010). 
31. Li, X. L., Yang, X., Hu, Y. X., Yu, X. D. \& Li, Q. L. A novel NAC transcription factor from Suaeda liaotungensis K. enhanced transgenic Arabidopsis drought, salt, and cold stress tolerance. Plant cell reports 33, 767-778, https://doi.org/10.1007/s00299-014-1602-y (2014).

32. He, L. et al. Mitochondrial Pyruvate Carriers Prevent Cadmium Toxicity by Sustaining the TCA Cycle and Glutathione Synthesis. Plant Physiol. 180, 198-211 (2019).

\section{Acknowledgements}

This research was supported by the Foundation for the National Key Research and Development Program of China grant (2017YFD0101801) to J.G., Shandong Upgraded Project of "Bohai Granary" Science and Technology Demonstration Engineering in 2019 "Collection of efficiency improvement technologies and Innovation of efficient green house agricultural models in severe saline-alkali land" to J.G., the Modern Agricultural Industrial Technology System Funding of Shandong Province grant (SDAIT-05-04) to J.G., the China Agriculture Research System (CARS-23-G14) to J.G., Distinguished Young Scholars of Shandong University grant (61200088963137) to W.Z. and Special Foundation for postdoctoral innovation projects grant (201603036) to L.H.

\section{Author contributions}

Ying Jing, Lin Shi, Lilong He performed the experiments with assistance from, Xin Li and Han Zheng; Lilong He and Ying Jing carried out most of the analyses with assistance from Mei Wang and Jianwei Gao; Wei Zhang and Lilong He designed the project and experiments; Lilong He and Wei Zhang wrote the manuscript. All authors read and approved the final manuscript.

\section{Competing interests}

The authors declare no competing interests.

\section{Additional information}

Supplementary information is available for this paper at https://doi.org/10.1038/s41598-019-56456-1.

Correspondence and requests for materials should be addressed to L.H. or W.Z.

Reprints and permissions information is available at www.nature.com/reprints.

Publisher's note Springer Nature remains neutral with regard to jurisdictional claims in published maps and institutional affiliations.

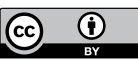

Open Access This article is licensed under a Creative Commons Attribution 4.0 International License, which permits use, sharing, adaptation, distribution and reproduction in any medium or format, as long as you give appropriate credit to the original author(s) and the source, provide a link to the Creative Commons license, and indicate if changes were made. The images or other third party material in this article are included in the article's Creative Commons license, unless indicated otherwise in a credit line to the material. If material is not included in the article's Creative Commons license and your intended use is not permitted by statutory regulation or exceeds the permitted use, you will need to obtain permission directly from the copyright holder. To view a copy of this license, visit http://creativecommons.org/licenses/by/4.0/.

(C) The Author(s) 2019 